\title{
Permeability of fractal porous media by Monte Carlo simulations
}

\author{
Boming $\mathrm{Yu}^{\mathrm{a}, \mathrm{b}, *}$, Mingqing Zou ${ }^{\mathrm{a}}$, Yongjin Feng ${ }^{\mathrm{a}}$ \\ ${ }^{a}$ Department of Physics and the State Key Laboratory of Plastic Forming and Die \& Mold Tech., \\ Huazhong University of Science and Technology, 1037 Luoyu Road, Wuhan, 430074, PR China \\ b The State Key Laboratory of Nonlinear Mechanics (LNM), Institute of Mechanics, Chinese Academy of Science, \\ Beijing 100080, PR China
}

Available online 7 April 2005

\begin{abstract}
The permeability of the fractal porous media is simulated by Monte Carlo technique in this work. Based on the fractal character of pore size distribution in porous media, the probability models for pore diameter and for permeability are derived. Taking the bi-dispersed fractal porous media as examples, the permeability calculations are performed by the present Monte Carlo method. The results show that the present simulations present a good agreement compared with the existing fractal analytical solution in the general interested porosity range. The proposed simulation method may have the potential in prediction of other transport properties (such as thermal conductivity, dispersion conductivity and electrical conductivity) in fractal porous media, both saturated and unsaturated.
\end{abstract}

(c) 2005 Elsevier Ltd. All rights reserved.

Keywords: Permeability; Fractal; Porous media; Monte Carlo simulation

\section{Introduction}

The permeabilities for porous media, both saturated and unsaturated, have received extensive attention [1$6]$ in the past decades. Since the microstructures of real porous media are usually disordered and extremely complicated, this makes it very difficult to analytically find the permeability of the media. Conventionally, the permeabilities of porous media were found by experiments $[1,2]$. In addition, much effort was devoted to numerical simulations such as the lattice gas (LG) [4] and Lattice Boltzmann method (LBM) $[5,6]$. However, the results

\footnotetext{
* Corresponding author. Tel.: +86 27 87542153; fax: +8627 87542153/45438.

E-mail address: yu3838@public.wh.hb.cn (B. Yu).
}

from either experiments or numerical simulations are usually expressed as correlations with one or more empirical constants or as curves, and the mechanisms behind the phenomena are thus often ignored. Therefore, seeking an analytical solution of permeability for porous media becomes a challenging task.

Fortunately, the porous media have been proved to be fractal objects in nature [7-14]. Katz and Thompson [7] may be the first to present the experimental evidence indicating that the pore spaces of a set of sandstone samples are fractals and are self-similar over 3-4 orders of magnitude in length extending from $10 \AA$ to $100 \mu \mathrm{m}$. They argued that the pore volume is a fractal with the same fractal dimension as the pore-rock interface. Krohn and Thompson [8] showed a set of sandstone pores and found that they are fractals and follow the 


\begin{tabular}{|c|c|c|c|}
\hline \multicolumn{4}{|c|}{ Nomenclature } \\
\hline$A$ & area of a unit cell & \multicolumn{2}{|c|}{ Greek symbols } \\
\hline$D_{\mathrm{f}}$ & pore area fractal dimension & $\sigma$ & variance \\
\hline$D_{\mathrm{T}}$ & fractal dimension of tortuous streamlines & $\phi$ & porosity \\
\hline$J$ & total number of Monte Carlo simulations & & pore size/diameter \\
\hline$K$ & $\begin{array}{l}\text { for one run for a porosity } \\
\text { permeability }\end{array}$ & $\mu$ & viscosity \\
\hline$L$ & length scale & \multicolumn{2}{|c|}{ Subscripts } \\
\hline$L_{0}$ & representative/straight length & $\mathrm{c}$ & cluster \\
\hline$N$ & $\begin{array}{l}\text { cumulative number of pores or total simula- } \\
\text { tion number for a converged permeability }\end{array}$ & $\begin{array}{l}\max \\
\min \end{array}$ & $\begin{array}{l}\operatorname{maximum} \\
\text { minimum }\end{array}$ \\
\hline$q$ & flow rate & $\mathrm{t}$ & total \\
\hline$Q$ & total flow rate & $\mathrm{p}$ & pore \\
\hline$R$ & $\begin{array}{l}\text { cumulative probability, defined by Eq. (7), } \\
\text { or random number defined by Eq. (10) }\end{array}$ & $i$ & $\begin{array}{l}\text { the } i \text { th capillary chosen randomly by Eq. } \\
\text { (10), or the } i \text { th simulation }\end{array}$ \\
\hline $\begin{array}{l}\bar{R}_{\mathrm{c}} \\
\langle\rangle\end{array}$ & $\begin{array}{l}\text { mean radius of clusters } \\
\text { means }\end{array}$ & & \\
\hline
\end{tabular}

fractal power laws. Smidt and Monro [10] performed experimental investigations on the images of laboratorymade synthetic sandstone and on modeled sandstone. Their results showed that the pore space of both the synthetic and the modeled sandstone was found to be fractal and the fractal scaling laws were obtained by the Box-counting method. This suggests that the laboratory synthetic stone pores are also fractal objects. For more evidences that the porous media are fractal objects, readers may consult the references [7-14].

Sahimi [3] reviewed and discussed theoretical and experimental approaches to flow, hydrodynamic dispersion, and miscible and immiscible displacement processes and their effective properties in reservoir rocks from continuum models to fractals, percolation, cellular automata. In his review, several empirical correlations for permeability are presented. However, these correlations are expressed as a function of only porosity with several empirical constants which have no physical meaning.Adler [15] concluded that the permeability in real porous media can be expressed as $K=K\left(\varepsilon, D_{\mathrm{f}}, \ldots\right)$ (where $\varepsilon$ and $D_{\mathrm{f}}$ are porosity and fractal dimension, respectively). However, no quantitative expression was given. Recently, $\mathrm{Yu}$ and Cheng [13] developed a fractal permeability model for bi-dispersed (saturated) porous media based on the fractal characteristics of pore sizes of the media, and this fractal model is also applicable to anisotropic porous fabrics $[14,16]$.

In this paper, the Monte Carlo simulations are performed based on the fractal natures of pore size distribution in porous media. The predictions from the present Monte Carlo simulations are compared with those from the existing analytical solution for bi-dispersed porous media, real porous media.

\section{Formulation of the Monte Carlo technique}

The pore microstructures, both the pore sizes and the pore-interfaces, of such porous media exhibit the fractal characteristics, and these media are called fractal porous media. The present work only deals with these media. The cumulative size-distribution of pores or islands (on earth) or spots (on engineering surfaces) whose sizes are greater than or equal to the size $\lambda$ have been proven to follow the fractal scaling law [12-14]:

$N(L \geqslant \lambda)=\left(\frac{\lambda_{\max }}{\lambda}\right)^{D_{\mathrm{f}}}$

where $D_{\mathrm{f}}$ is the pore area fractal dimension, $1<D_{\mathrm{f}}<2$ in two dimensions and $\lambda_{\max }$ is the maximum pore size. Eq. (1) implies that there is only one maximum pore in a porous medium or one maximum island on earth, and this is consistent with the practical situation.

Differentiating equation (1) with respect to $\lambda$ results in the number of pores whose sizes are within the infinitesimal range $\lambda$ to $\lambda+\mathrm{d} \lambda$,

$-\mathrm{d} N=D_{\mathrm{f}} \lambda_{\max }^{D_{\mathrm{f}}} \lambda^{-\left(D_{\mathrm{f}}+1\right)} \mathrm{d} \lambda$

where $\mathrm{d} \lambda>0$. The negative sign in Eq. (2) implies that the island or pore number decreases with the increase of island or pore size, and $-\mathrm{d} N>0$. Eq. (1) describes the scaling relationship of the cumulative pore population. The total number of pores or islands or spots, from the smallest diameter $\lambda_{\min }$ to the largest diameter $\lambda_{\max }$, can be obtained from Eq. (1) as $[12,13]$

$N_{t}\left(L \geqslant \lambda_{\min }\right)=\left(\frac{\lambda_{\max }}{\lambda_{\min }}\right)^{D_{\mathrm{f}}}$ 
Dividing Eq. (2) by Eq. (3) gives

$-\frac{\mathrm{d} N}{N_{t}}=D_{\mathrm{f}} \lambda_{\min }^{D_{\mathrm{f}}} \lambda^{-\left(D_{\mathrm{f}}+1\right)} \mathrm{d} \lambda=f(\lambda) \mathrm{d} \lambda$

where $f(\lambda)=D_{\mathrm{f}} \lambda_{\min }^{D_{\mathrm{f}}} \lambda^{-\left(D_{\mathrm{f}}+1\right)}$ is the probability density function and $f(\lambda) \geqslant 0$. Patterned after the probability theory, the probability density function $f(\lambda)$ should satisfy the following normalization relationship or total cumulative probability:

$\int_{-\infty}^{\infty} f(\lambda) \mathrm{d} \lambda=\int_{\lambda_{\min }}^{\lambda_{\max }} f(\lambda) \mathrm{d} \lambda=1-\left(\frac{\lambda_{\min }}{\lambda_{\max }}\right)^{D_{\mathrm{f}}} \equiv 1$

The integration result of Eq. (5) shows that Eq. (5) holds if and only if

$$
\left(\frac{\lambda_{\min }}{\lambda_{\max }}\right)^{D_{\mathrm{f}}} \cong 0
$$

is satisfied. Eq. (6) implies that $\lambda_{\min } \ll \lambda_{\max }$ must be satisfied for fractal analysis of a porous medium, otherwise the porous medium is a non-fractal medium, and Eq. (6) can be considered as a criterion [12] whether a porous medium can be characterized by fractal theory and technique. In general, $\lambda_{\min } / \lambda_{\max }<10^{-2}$ in porous media and Eq. (6) holds approximately, thus the fractal theory and technique can be used to analyze properties of porous media.

The cumulative probability $(R)$ in the range of $\lambda_{\min } \sim \lambda$ can be found by

$$
\begin{aligned}
R(\lambda) & =\int_{\lambda_{\min }}^{\lambda} f(\lambda) \mathrm{d} \lambda=\int_{\lambda_{\min }}^{\lambda} D_{\mathrm{f}} \lambda_{\min }^{D_{\mathrm{f}}} \lambda^{-\left(D_{\mathrm{f}}+1\right)} \mathrm{d} \lambda \\
& =1-\left(\frac{\lambda_{\min }}{\lambda}\right)^{D_{\mathrm{f}}}
\end{aligned}
$$

Eq. (7) indicates that $R=0$ as $\lambda \rightarrow \lambda_{\min }$ and $R \approx 1$ as $\lambda \rightarrow \lambda_{\max }$. The latter case is expected because of Eq. (6). Since the pore size $\lambda$ in a porous medium is randomly distributed in the range of $\lambda_{\min } \sim \lambda_{\max }, R$ in Eq. (7) is in the range of $0-1$ and thus approximately corresponds to a set of random numbers of $0-1$.

From Eq. (7), we can obtain

$1-R=\left(\frac{\lambda_{\min }}{\lambda}\right)^{D_{\mathrm{f}}}$

From Eq. (8), a pore size $\lambda$ is expressed as

$\lambda=\frac{\lambda_{\min }}{(1-R)^{1 / D_{\mathrm{f}}}}=\left(\frac{\lambda_{\min }}{\lambda_{\max }}\right) \frac{\lambda_{\max }}{(1-R)^{1 / D_{\mathrm{f}}}}$

where $\lambda_{\min } \leqslant \lambda \leqslant \lambda_{\max }$. Eq. (9) is a probability model for pore size in the present simulation. For the $i$ th capillary tube chosen randomly, Eq. (9) can be written as

$\lambda_{i}=\frac{\lambda_{\min }}{\left(1-R_{i}\right)^{1 / D_{\mathrm{f}}}}=\left(\frac{\lambda_{\min }}{\lambda_{\max }}\right) \frac{\lambda_{\max }}{\left(1-R_{i}\right)^{1 / D_{\mathrm{f}}}}$

where $i=1,2,3, \ldots, J$, and $J$ is the total number of Monte Carlo simulations in one run for a given porosity. Eq. (9) or (10) presents an explicit model for pore size distribution in porous media, whereas an analytical solution such as Ref. [13] cannot provide such an explicit model for pore size distribution. Eq. (9) or (10) may be helpful for one to understand the meaning of Monte Carlo simulations. Eq. (10) denotes that since $R_{i}$ is a random number of $0-1$ produced by computer, thus, the pore size $\lambda_{i}$ is determined randomly, and this also simulates the randomness and fractal distribution of pore sizes in porous media because Eq. (10) is based on Eq. (1). This mechanism can be easily understood if one carefully examines, for instance, a sample of soil, one can easily find that the pores with different sizes are randomly distributed in the sample, not regularly distributed in the sample. This means that the pore sizes are not only randomly distributed but also follow the fractal power law Eq. (1). Therefore, we can choose random number of $R$ and determine the pore sizes by Eq. (10) if $\lambda_{\min }$ or $\lambda_{\min } / \lambda_{\max }$ and $\lambda_{\max }$ is known for a porous medium.

In Eq. (10) the pore area fractal dimension $D_{\mathrm{f}}$ for saturated porous media is given by [12]

$D_{\mathrm{f}}=d-\frac{\ln \phi}{\ln \frac{\lambda_{\min }}{\lambda_{\max }}}$

where $\phi$ is the effective porosity of porous media, $d$ is the Euclidean dimension, and $d=2$ and 3 in the two- and three-dimensional spaces, respectively. Eq. (11) exactly holds for exactly self-similar fractal geometries and approximately holds for random or disordered fractal porous media. Eq. (11) implies that the statistical selfsimilarity exists in the range of $\lambda_{\min } \sim \lambda_{\max }$ for porous media.

In this work, both the fractal distribution and tortuosity of capillary tubes in porous media are included. Now, let the diameter of a capillary in the medium be $\lambda$ and its tortuous length along the flow direction be $L_{t}(\lambda)$. Due to the tortuous nature of the capillary, $L_{t}(\lambda) \geqslant L_{0}$, with $L_{0}$ being the representative length. For a straight capillary, $L_{t}(\lambda)=L_{0}$, The relationship between the diameter and length of capillaries exhibits the fractal scaling law $[13,14]$ :

$L_{t}(\lambda)=\lambda^{1-D_{\mathrm{T}}} L_{0}^{D_{\mathrm{T}}}$

where $D_{\mathrm{T}}$ is the tortuosity fractal dimension with $1<D_{\mathrm{T}}<2$ in two dimensions, representing the extent of convolutedness of capillary pathways for fluid flow through a medium.

The flow rate through a single tortuous capillary is given by modifying the well known Hagen-Poiseulle equation [17] to give

$q(\lambda)=G \frac{\Delta P}{L_{t}(\lambda)} \frac{\lambda^{4}}{\mu}$

where $G=\pi / 128$ is the geometry factor for flow through a circular capillary, $\mu$ is the viscosity of the fluid, $\Delta P$ is 
the pressure gradient. Thus, the total flow rate $Q$ for flow through a unit cell with total cross-sectional area $A$ can be obtained by adding the individual flow rates, $q\left(\lambda_{i}\right)$. Due to Eqs. (12) and (13), we have

$$
\begin{aligned}
Q & =\sum_{i=1}^{J} q\left(\lambda_{i}\right)=\sum_{i=1}^{J} G\left(\frac{\Delta P}{L_{0}} \frac{A}{\mu}\right) \frac{L_{0}^{1-D_{\mathrm{T}}}}{A} \lambda_{i}^{3+D_{\mathrm{T}}} \\
& =G\left(\frac{\Delta P}{L_{0}} \frac{A}{\mu}\right) \frac{L_{0}^{1-D_{\mathrm{T}}}}{A} \sum_{i=1}^{J} \lambda_{i}^{3+D_{\mathrm{T}}}
\end{aligned}
$$

where $L_{0}$ is the representative length of a unit cell and approximated by

$L_{0}=\sqrt{A}$

Eq. (15) implies that a cubic sample is applied.

Comparing to Darcy's law, we obtain the permeability expression as follows:

$K=G \frac{L_{0}^{1-D_{\mathrm{T}}}}{A} \sum_{i=1}^{J} \lambda_{i}^{3+D_{\mathrm{T}}}$

Due to Eqs. (10) and (15), Eq. (16) can be rewritten as

$$
\begin{aligned}
K= & G A^{-\left(1+D_{\mathrm{T}}\right) / 2}\left(\frac{\lambda_{\min }}{\lambda_{\max }}\right)^{3+D_{\mathrm{T}}} \\
& \times \lambda_{\max }^{3+D_{\mathrm{T}}} \sum_{i=1}^{J} \frac{1}{\left(1-R_{i}\right)^{\left(3+D_{\mathrm{T}}\right) / D_{\mathrm{f}}}}
\end{aligned}
$$

Eq. (17) is the probability model for effective permeability. Eq. (17) indicates that the permeability is a function of parameters $\mathrm{A}, \lambda_{\min }, \lambda_{\max }, D_{\mathrm{T}}, D_{\mathrm{f}}$ and random number $R_{i}$. Once the parameters $A, \lambda_{\min }, \lambda_{\max }, D_{\mathrm{T}}$ and $D_{\mathrm{f}}$ are determined, the permeability $K$ can be calculated by Monte Carlo simulation through choosing a set of random numbers, $R_{i}, i=1,2,3, \ldots, J$.

The total cross sectional area $A$ (in Eq. (17)) of a unit cell is related to porosity by

$A=A_{\mathrm{p}} / \phi$

where $A_{\mathrm{p}}$ is the total pore area in a unit cell and determined by

$A_{\mathrm{p}}=\sum_{i=1}^{J} a_{i}=\sum_{i=1}^{J} \pi \lambda_{i}^{2} / 4$

where $a_{i}$ is the area of cross section of the $i$ th capillary tube chosen by Monte Carlo simulation through Eq. (10). It can be seen that the total cross sectional area $A$ in this model can be determined by Eqs. (18) and (19) by the Monte-Carlo technique if porosity $\phi$ is given.

In Eq. (17), $\lambda_{\min }$ and $\lambda_{\max }$ are dependent upon a particular porous medium and can be measured by image analysis of a sample, and $D_{\mathrm{f}}$ is calculated by Eq. (11) or measured by the box-counting method [3,13]. The fractal dimension $D_{\mathrm{T}}$ for tortuous streamlines is usually determined by the box-counting method or Monte Carlo simulation, and an analytical expression for $D_{\mathrm{T}}$ has not yet been developed. Using the Monte Carlo simulation, Wheatcraft and Tyler [18] obtained the fractal dimension $D_{\mathrm{T}}=1.087$ for flow through heterogeneous media. Applying the box-counting method to measure a set of possible streamlines in a porous medium, Yu and Cheng [13] obtained the fractal dimension $D_{\mathrm{T}}=1.10$ for flow through the bi-dispersed porous media. We again use $D_{\mathrm{T}}=1.10$ for calculation of permeability in this work.

It is worth pointing out that the present model is also based on the assumption that the porous medium consists of a set/bundle of parallel and tortuous capillaries/ tubes with uniform diameters. Although the tortuous capillaries/tubes are not uniform in real porous media, the results by Shih and Lee [19] showed that the permeability of a real capillary with non-uniform diameter can be considered as a series of capillaries with different diameters and the permeability is mainly determined by the smallest capillary. This means that the permeability is approximately determined by the smallest diameter of a tortuous non-uniform capillary. This also means that the permeability for flow through a real non-uniform capillary is equivalent to that for flow thorough a uniform capillary with the smallest diameter. On the other hand, since the statistical self-similarity exists in every porous section perpendicular to flow direction, the permeability for flow through every porous section should be the same and is independent of a section chosen. From the above discussions, it is seen that the present model, based on the assumption that the porous medium consists of a set/bundle of parallel and tortuous capillaries/ tubes with uniform diameters, is an acceptable approximation to real porous media.

\section{Results and discussions}

\subsection{Algorithm for permeability}

In the following, we take the bi-dispersed porous media as examples of real porous media to demonstrate how to apply the proposed technique to calculate the permeability of porous media.

The bi-dispersed porous medium is composed of clusters, which are agglomerated by small particles. There are macro-pores and micro-pores between and within the clusters, respectively, see Fig. 1(a). Fig. 1(b) demonstrates a typical fractal scaling law for the medium. The pore area fractal dimension, $D_{\mathrm{f}}$, can be determined by the value of the slope of a linear fit through the data on a $\log -\log$ plot of the cumulative number of box (pore) number $N(L \geqslant \lambda$ ) versus the box (pore) size $\lambda$ based on Eq. (1). This proves that the bi-dispersed porous media are fractal objects. Since the clusters and particles within the clusters are randomly distributed, the macro-pores and micro-pores are also randomly distributed in bi-dispersed porous media. 

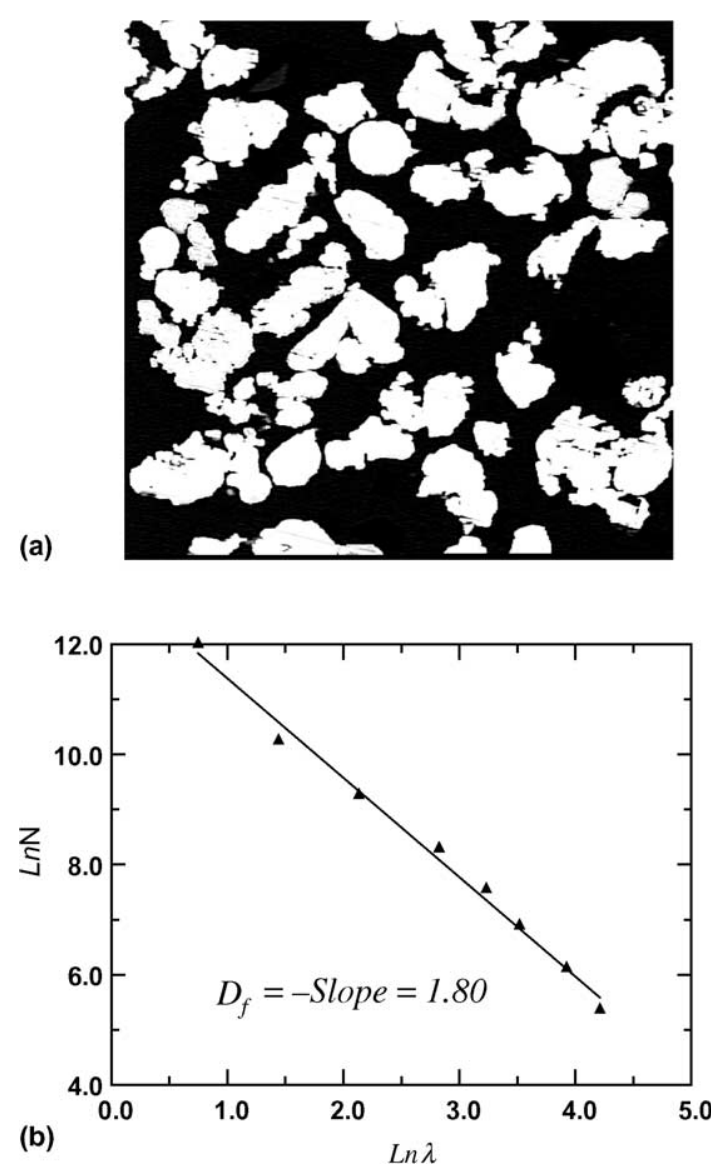

Fig. 1. (a) An image photo [13] of the bi-dispersed porous medium $(\phi=0.54)$ at magnification of 50 , the white are solid and the black are pores (since the micro-pores inside clusters are very small and the cooper particles are soft, it is difficult to see the micro-pores inside clusters after the sample being polished.), and (b) the cumulative pore number $N(L \geqslant \lambda)$ versus $\lambda$.

For the bi-dispersed porous media, $\mathrm{Yu}$ and Cheng have obtained [13],

$\frac{\lambda_{\min }}{\lambda_{\max }}=\frac{\sqrt{2}}{d^{+}} \sqrt{\frac{1-\phi}{1-\phi_{\mathrm{c}}}}$

where $d^{+}=24$ is the ratio of the cluster mean size to the minimum particle size, and $\phi_{\mathrm{c}}$ is the micro porosity in the cluster and given by [20]

$\phi_{\mathrm{c}}=0.342 \phi$

The average maximum pore size is given by [13]

$\lambda_{\text {max }}=\frac{\bar{R}_{\mathrm{c}}}{2}\left[\sqrt{2\left(\frac{1-\phi_{\mathrm{c}}}{1-\phi}-1\right)}+\sqrt{\frac{2 \pi}{\sqrt{3}} \frac{1-\phi_{\mathrm{c}}}{1-\phi}}-2\right]$

where $\bar{R}_{\mathrm{c}}=0.30 \mathrm{~mm}$ [13] is the cluster mean radius. The total area of a unit cell for the medium is [13]
$A=\frac{1}{2} \pi \bar{R}_{\mathrm{c}}^{2} \frac{1-\phi_{\mathrm{c}}}{1-\phi}$

The algorithm for determination of the permeability of a bi-dispersed porous medium is summarized as follows:

1. Given a porosity $\phi$, find the micro porosity $\phi_{\mathrm{c}}$ in cluster from Eq. (21).

2. Find $\lambda_{\min } / \lambda_{\max }, \lambda_{\max }$ and total area $A$ of a unit cell from Eqs. (20), (22) and (23), respectively, or from an image analysis of the sample.

3. Find pore area fractal dimension $D_{\mathrm{f}}$ from Eq. (11) (let $d=2$ ), or from the box-counting method.

4. Produce a random number $R_{i}$ of $0-1$ by the MonteCarlo method.

5. Calculate $\lambda_{i}$ by Eqs. (10), (20) and (22).

6. If $\lambda_{i}>\lambda_{\max }$, return to procedure 4 , otherwise continue to the next procedure.

7. Find $A_{\mathrm{p}}$ from Eq. (19).

8. Calculate the permeability $K$ from Eq. (16) or (17) (with $D_{\mathrm{T}}=1.10$ [13], or $D_{\mathrm{T}}$ measured from the boxcounting method).

Procedures 5-8 are repeated for calculation of permeability until a converged value is obtained at a given porosity. Procedure 6 means that the randomly produced pore size $\lambda_{i}$ in Monte Carlo simulation is not allowed to exceed the maximum pore size $\lambda_{\max }$ in order to coincide with physical situation. Since the existing analysis [21] showed that the smaller scale/tiny pores in porous media play the negligible effect on the total/effective permeability, no restriction is imposed on the minimum value of pore size $\lambda_{i}$ in Eq. (10) in this simulation.

The convergence creterion is that when the following condiction is satistied, i.e.

$A_{J}=A_{\mathrm{p}} / \phi>A$

stop the simulation and record the final/convergent permeability and the total simulated number $(J)$ in one run for a given porosity. In Eq. (24), $A_{J}$ is the total area calculated after the $J$ th computation in one run and $A$ is given by Eq. (23). If the converged permeability is obtained in one run, set the permeability as $K_{n}$ $(n=1,2,3, \ldots, N)$. Then the averaged permeability for a given porosity can be calculated by

$$
\langle K\rangle=\frac{1}{N} \sum_{n=1}^{N} K_{n}
$$

where $N$ is the total number of runs for a given porosity.

The variance is defined by

$\sigma=\sqrt{\left\langle K^{2}\right\rangle-\langle K\rangle^{2}}$ 
where

$\left\langle K^{2}\right\rangle=\frac{1}{N} \sum_{n=1}^{N} K_{n}^{2}$

From the above algorithm for determination of the permeability by this method, it is found that the algorithm is quite simple, and the calculation of permeability for 100,000 runs takes only several minites in a microcomputer. No grid generation, no discretization, no boundary conditions and no calculations of fluid field are required for permeability by the present method, whereas the conventional numerical methods such as the LG and LBM are based on a discrete particle kinetics utilizing a discrete lattice and discrete time. The LBM can also be viewed as a special finite difference scheme for the kinetic equation of the discrete-velocity distribution function. The numerical accuracy of the LG and LBM is usually determined by boundary treatments, Mach number [4]. The second advantage of the present model over the conventional numerical methods is that the present model is explicitly expressed as a function of microstructural parameters, $D_{\mathrm{f}}$ and $D_{\mathrm{T}}$, total area $A$ of a unit cell, maximum pore size $\lambda_{\max }$, minimum pore size $\lambda_{\min }$ as well as random number $R_{i}$. So, the mechanisms affecting the permeability are better understood. While the conventional numerical methods cannot explicitly express the permeability as a function of microstructural parameters. The third advantage of the present model over the conventional numerical method is that the conventional numerical methods such as the LG and LBM require to solve a set of discrete NavierStokes equations for velocity field for flow through artificially generated complex porous medium, so the computation is usually time-consuming. Therefore, the advantages of the present method over the conventional numerical methods are evident. However, the above comparisons do not imply that the present method is an alternative/replacement to these modeling approaches such as the LG and LBM.

\subsection{Results and discussions}

Due to Eqs. (20) and (22), the minimum pore size in this example can be estimated by

$$
\begin{aligned}
\lambda_{\min }= & \left(\frac{\lambda_{\min }}{\lambda_{\max }}\right) \lambda_{\max } \\
= & \frac{\sqrt{2} \bar{R}_{\mathrm{c}}}{2 d^{+}}\left[\sqrt{2\left(\frac{1-\phi_{\mathrm{c}}}{1-\phi}-1\right)}\right. \\
& +\sqrt{\left.\frac{2 \pi}{\sqrt{3}} \frac{1-\phi_{\mathrm{c}}}{1-\phi}-2\right] \sqrt{\frac{1-\phi}{1-\phi_{\mathrm{c}}}}}
\end{aligned}
$$

Fig. 2 gives the pore sizes randomly chosen by the probability model Eq. (10) for pore size in the present simulations under the given parameters Eqs. (20) and

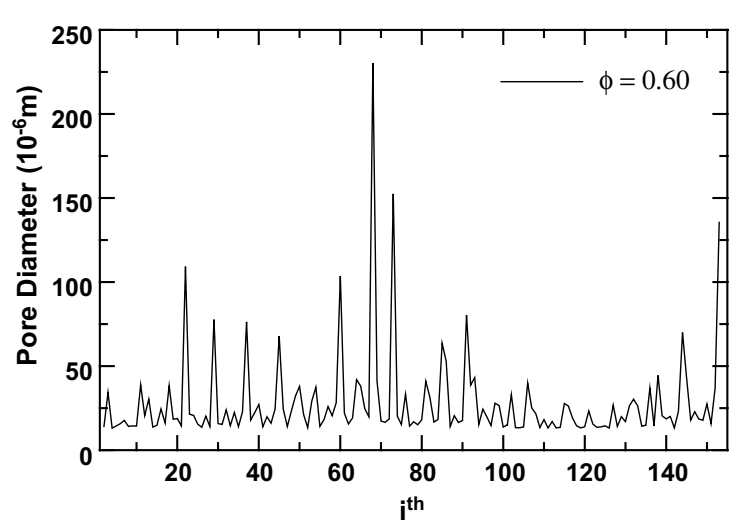

Fig. 2. The pore sizes simulated by the present Monte Carlo simulations.

(22) or Eq. (27) for bi-dispersed porous media. From Fig. 2 it is also seen that the number of larger pores is much less than that of smaller pores, this is qualitatively consistent with fractal theory. It is also found from Fig. 2 that the minimum pore size is about $13 \mu \mathrm{m}$. Estimation from Eq. (27) gives $\lambda_{\text {min }}=13.1 \mu \mathrm{m}$ at porosity 0.60 . This shows that the probability model Eq. (10) for pore size is correct.

Fig. 3 presents the effective permeabilities by the present Monte Carlo technique in 1000 runs of simulations at porosity 0.60 . From Fig. 3 it can be seen that the simulated permeabilities roughly fluctuate around the permeability of 600 Darcy. This is exactly expected because of the random character of pore size distribution. But the averaged permeability can be estimated to be about 600 Darcy at porosity 0.60 . Table 1 lists the effective permeabilities and variances in different runs at different porosities in the present Monte Carlo simulations. Form Table 1 it can be seen that the converged permeability is reached when the number of total runs is greater than 1000 . Since the averaged permeability at $N=1000$

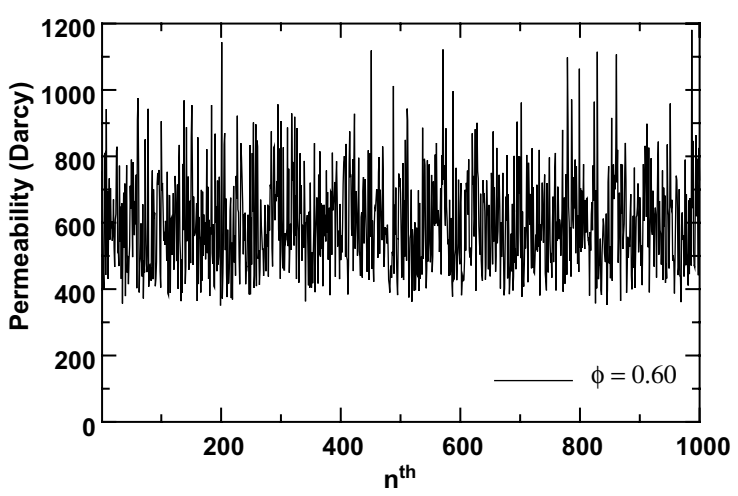

Fig. 3. The simulated permeabilities by the Monte Carlo technique in 1000 runs at porosity 0.60 . 
Table 1

The simulated permeabilities (Darcy) by the present Monte Carlo technique in different numbers $(N)$ of runs of simulations at different porosities

\begin{tabular}{llllll}
\hline Porosity & $N=100$ & $N=1000$ & $N=10,000$ & $N=100,000$ & Variance $\sigma$ as $N=100,000$ \\
\hline 0.60 & 641.4 & 600.0 & 599.4 & 597.6 & 153.9 \\
0.55 & 386.9 & 405.3 & 393.8 & 394.6 & 103.1 \\
0.50 & 244.1 & 263.8 & 267.0 & 263.1 & 68.5 \\
0.45 & 166.8 & 174.7 & 176.9 & 175.0 & 46.2 \\
\hline
\end{tabular}

is almost the same as that at $N=100,000$, the variances $\sigma$ are only given at $N=100,000$. It is seen that the variance is larger and is about $26 \%$ of averaged permeability. This reveals that permeability fluctuations are rough, and this can be also seen from Figs. 2 and 3. This is consistent with the physical situation because in a real random porous media some pores may be very large, which contribute larger permeabilities, while some other pores may be very small, which contribute relatively smaller permeabilities. It is evident that the analytical solution cannot reveal such a physical phenomenon.

From Figs. 2 and 3, it is also see that the present Monte Carlo simulation can provide the random distribution character of pores and the fluctuation character of permeability, based on the proposed probability models Eq. (10) for pore diameters. Although the pores are randomly chosen and the rough oscillations/fluctuations of the permeabilities are observed, the converged permeabilities still can be obtained as long as the enough number of runs of simulations is performed. Figs. 2 and 3 , therefore, may be helpful for one to understand the essence of Monte Carlo simulation on permeability. But, an analytical model such as Ref. [13] cannot provide such information, and the analytical model can only provide a continuum/smooth curve $f(\lambda) \sim \lambda$ and the final permeability result such as the solid line in Fig. 4.

Fig. 4 compares the permeabilities by the present Monte Carlo technique with those from the existing fractal analytical solution. It is shown that they are in good agreement. The deviations between the simulated results by the Monte Carlo method and those by fractal analysis are small. This verifies the validity of the present simulation technique.

Fig. 5 illustrates the effect of the fractal dimension $D_{\mathrm{T}}$ on permeability. The figure shows that permeability decreases with the increase of the fractal dimension $D_{\mathrm{T}}$. This is expected because the higher the fractal dimension $D_{\mathrm{T}}$, the higher the tortuosity of capillaries, causing the higher resistance to flow in porous media and thus the lower permeability. In the limiting case of $D_{\mathrm{T}}=2$, we have so highly tortuous capillaries that fill a two-dimensional plane. This situation corresponds to the case of lowest possible value of permeability, which is consistent with the real physical situation. Fig. 5 also reveals that when $D_{\mathrm{T}}=1$ (corresponding to the case of straight capillary), the permeability is higher about $10 \%$ than

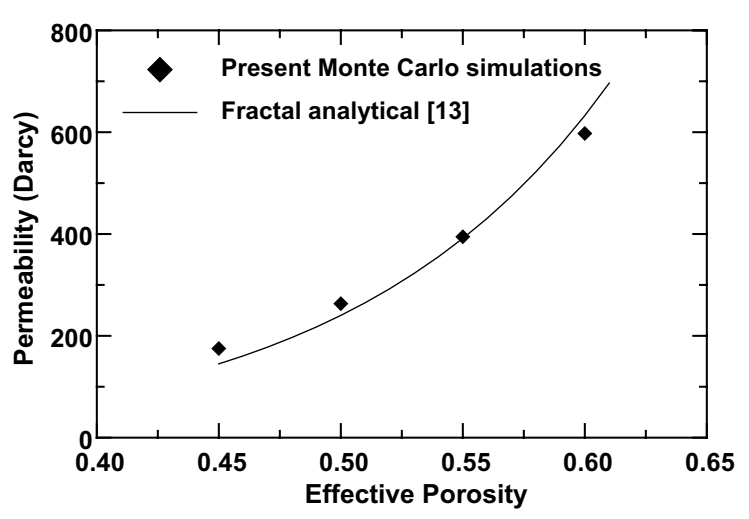

Fig. 4. A comparison of the permeabilities predicted by the present Monte Carlo simulations (at $D_{\mathrm{T}}=1.10$ ) those obtained by the fractal analytical solution [13].

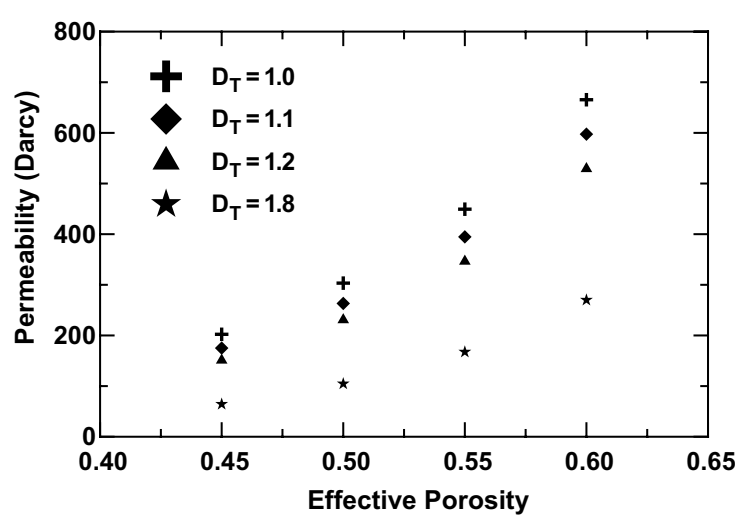

Fig. 5. The effect of tortuosity fractal dimension $D_{\mathrm{T}}$ permeability.

that when $D_{\mathrm{T}}=1.1$, and when $D_{\mathrm{T}}=1.2$, the permeability is lower about $10 \%$ comparing to that as $D_{\mathrm{T}}=1.1$. At high tortuosity $D_{\mathrm{T}}=1.8$, the permeability is lower about $120 \%$ comparing to that as $D_{\mathrm{T}}=1.1$. This means that tortuosity should be taken into account if accurate permeability is desired. In this work, $D_{\mathrm{T}}=1.1$ (obtained by the Box-counting method [13]) is again used to calculate the permeability for comparison with analytical permeability [13] (see Fig. 4). It might be better, if an analytical tortuosity fractal dimension $D_{\mathrm{T}}$ is applied. However, 
since the analytical permeability was based on the tortuosity fractal dimension $D_{\mathrm{T}}=1.1$ obtained by the Boxcounting method, this work only compares the present MC results with the analytical results based on $D_{\mathrm{T}}=$ 1.1. A recent report [22] on the analytical tortuosity fractal dimension $D_{\mathrm{T}}$ shows that the tortuosity fractal dimension $D_{\mathrm{T}}$ is indeed about 1.10 in the porosity range of $0.45-0.60$. For an analytical model for the tortuosity fractal dimension $D_{\mathrm{T}}$, readers may consult Ref. [22].

\section{Concluding remarks}

The Monte Carlo technique is applied to predict the permeability of fractal porous media. The probability models for pore distribution and for permeability are expressed as a function of fractal dimensions, $D_{\mathrm{f}}, D_{\mathrm{T}}$, total area $A$ of a sample or unit cell, maximum pore size $\lambda_{\text {max }}$, minimum pore size $\lambda_{\text {min }}$ as well as random number $R_{i}$. There is no empirical constant in the proposed models and every parameter in the models has clear physical meaning. The validity of the present simulations is verified by a comparison with existing analytical solution for permeability of the bi-dispersed porous media.

It is worth pointing out that if a porous medium is a single-scale fractal medium, the permeability can be easily found by performing an integral analytically. However, if a porous medium is a multiscale or scaledependent fractal medium, the parameters $\lambda_{\min }, \lambda_{\max }$, $D_{\mathrm{f}}$ and $D_{\mathrm{T}}$ are related to the scales $\lambda$, in this case, the integral may not be performed analytically and we cannot obtain an analytical expression for permeability. For this situation, the numerical integral may need to be carried out for permeability, and the Monte Carlo technique might be one of the possible numerical methods. Then, we can still obtain an explicit expression for permeability in this case, no matter whether a porous medium is a single-scale or multiscale or scale-dependent fractal medium. In addition, the present technique may also have the general interests/potentials in analysis of other transports such as thermal, electrical in fractal porous media. Therefore, the proposed technique may provide us with a new approach in addition to the analytical and other numerical methods.

\section{Acknowledgement}

The authors would like to acknowledge the support of this work by the National Natural Science Foundation of China through Grant no. 10272052.

\section{References}

[1] J. Bear, Dynamics of Fluids in Porous Media, American Elsevier Publishing Company, Inc., 1972.
[2] M. Kaviany, Principles of Heat Transfer in Porous Media, second ed., Springer-Verlag, Inc., New York, 1995.

[3] M. Sahimi, Flow phenomena in rock: from continuum models to fractals percolation, cellular automata, and simulated annealing, Rev. Mod. Phys. 65 (1993) 13931534.

[4] A. Koponen, M. Kataja, J. Timonen, Permeability and effective porosity of porous media, Phys. Rev. E 56 (1997) 3319-3325.

[5] S. Chen, G.D. Doolen, Lattice Boltzmann method for fluid flows, Annu. Rev. Fluid Mech. 30 (1998) 329-364.

[6] R.R. Nourgaliev, T.N. Dinh, T.G. Theofanous, D. Joseph, The lattice Boltzmann equation method: theoretical interpretation, numerics and implications, Int. J. Multiphase Flow 29 (2003) 117-169.

[7] A.J. Katz, A.H. Thompson, Fractal sandstone pores: Implications for conductivity and pore formation, Phys. Rev. Lett. 54 (1985) 1325-1328.

[8] C.E. Krohn, A.H. Thompson, Fractal sandstone pores: Automated measurements using scanning-electron-microscope images, Phys. Rev. B 33 (1986) 6366-6374.

[9] I.M. Young, J.W. Crawford, The fractal structure of soil aggregations: its measurement and interpretation, J. Soil Sci. 42 (1991) 187-192.

[10] J.M. Smidt, D.M. Monro, Fractal modeling applied to reservoir characterization and flow simulation, Fractals 6 (1998) 401-408.

[11] B.M. Yu, L.J. Lee, H.Q. Cao, Fractal characters of pore microstructures of textile fabrics, Fractals 9 (2001) 155163.

[12] B.M. Yu, J.H. Li, Some fractal characters of porous media, Fractals 9 (2001) 365-372.

[13] B.M. Yu, P. Cheng, A fractal permeability model for bidispersed porous media, Int. J. Heat Mass Transfer 45 (2002) 2983-2993.

[14] B.M. Yu, L.J. Lee, H.Q. Cao, A fractal in-plane permeability model for fabrics, Polym. Compos. 23 (2002) 201221.

[15] P.M. Adler, Transports in fractal porous media, J. Hydrol. 187 (1996) 195-213.

[16] B.M. Yu, J.H. Li, D.M. Zhang, A fractal trans-plane permeability model for textile fabrics, Int. Comm. Heat Mass Transfer 30 (2003) 127-138.

[17] M.M. Denn, Process Fluid Mechanics, Prentice Hall, NJ, 1980.

[18] S.W. Wheatcraft, S.W. Tyler, An explanation of scaledependent dispersivity in heterogeneous aquifers using concepts of fractal geometry, Water Resour. Res. 24 (1988) 566-578.

[19] C.-H. Shih, L.J. Lee, Effect of fiber architechture on permeability in liquid composite molding, Polym. Compos. 19 (1998) 626-639.

[20] Z.Q. Chen, P. Cheng, C.T. Hus, A theoretical and experimental study on stagnant thermal conductivity of bi-dispersed porous media, Int. Comm. Heat Mass Transfer 27 (2000) 601-610.

[21] B.M. Yu, L.J. Lee, A simplified in-plane permeability model for textile fabrics, Polym. Compos. 21 (2000) 660685.

[22] B.M. Yu, Fractal dimension for tortuous stream-tubes in porous media, Chin. Phys. Lett. 22 (2005) 158-160. 